\title{
Dichoptic temporal frequency differences do not lead to binocular rivalry
}

\author{
ROBERT P. O'SHEA and RANDOLPH BLAKE \\ Cresap Neuroscience Laboratory, Northwestern University, Evanston, Illinois
}

\begin{abstract}
The influence of temporal modulation on binocular rivalry was examined in two ways. First, an uncontoured field viewed by one eye had its luminance sinusoidally modulated at $4 \mathrm{~Hz}$ (fullfield flicker). The other eye viewed a similar field that flickered at different temporal frequencies ranging from .5 to $16 \mathrm{~Hz}$. Second, orthogonal sinusoidal dichoptic gratings were phase shifted so as to duplicate the range of temporal-frequency disparities presented with full-field flicker. Three subjects recorded exclusive visibility of the rival fields over 60-sec trials. Full-field flicker produced very few reports of rivalry, and was described as resembling "visual beats" or as an intermediate flicker rate. Usual amounts of rivalry were reported between orthogonal sine-wave gratings; the field flickering at low rates dominated less. These results were essentially confirmed in a second experiment, designed to minimize individual differences in response criteria by requiring subjects to respond to disappearances of small, dichoptic, nonrival markers superimposed on the rival fields. The markers disappeared in concert with episodes of rivalry suppression of the gratings, although disappearances were slightly briefer than supression durations. Disappearances of markers on full-field flicker were rare, extremely brief, and could be attributed to Troxler's fading rather than rivalry. The absence of rivalry between temporally disparate fullfield flicker suggests that rival interactions within transient mechanisms are minimal.
\end{abstract}

Something remarkable happens when the binocular visual system receives discrepant information from the two eyes: portions of one eye's view are suppressed for several seconds at a time, with these periods of suppression alternating between the two eyes. This breakdown in stable single vision, called binocular rivalry, is readily experienced when the two eyes view dissimilar monocular spatial patterns, such as orthogonally oriented contours. However, discrepant spatial information is not the only condition that instigates binocular rivalry. Identical spatial patterns may engage in vigorous rivalry if the monocular patterns move in different directions or at different velocities (Blake, Zimba, \& Williams, 1985; Fox, Todd, \& Bettinger, 1975; Wade, De Weert, \& Swanston, 1984), or if the patterns are different in color (Hollins \& Leung, 1978) or in brightness (Wallach \& Adams, 1954).

Is there any form of monocular image discrepancy (aside from retinal disparity) that can be tolerated by the binocular visual system? Suppose, for instance, that the eyes view two uncontoured flickering fields whose rates of flicker differ for each eye. For several reasons, we anticipated that interocular discrepancy in temporal frequency would trigger rivalry. First, it has long been known that temporal modulation (e.g., motion) of spatial patterns strongly influences the time-course of binocular rivalry (Breese, 1899): either movement or flicker of

This research was supported by Grant NSF 8418731 awarded to Randolph Blake. Address reprint requests to Robert O'Shea, Cresap Neuroscience Laboratory, 2021 Sheridan Rd., Evanston, IL 60201. one eye's rival target increases the predominance of that target relative to its stationary counterpart seen by the other eye. Second, Hollins (1980) reported that the spatial frequency at which rivalry is most clear-cut is that at which the putative transient channel is most sensitive (e.g., Kulikowski \& Tolhurst, 1973). On the basis of this finding, Hollins surmised that transient channels in human vision play a crucial role in binocular rivalry.

For these reasons, we anticipated that temporal frequency disparity between the two eyes would trigger rivalry. We were surprised, therefore, to observe no hint of alternating dominance when uncontoured fields flickering at different rates were viewed dichoptically. Instead, the resulting binocular percept resembled more or less a combination of the two fields. Intrigued by this observation and its implications for neural binocular integration, we quantitatively examined this phenomenon over a range of temporal frequencies.

\section{EXPERIMENT 1}

\section{Method}

Subjects. Three subjects participated, all of whom had corrected or uncorrected Snellen acuity of 6/6 or better in each eye and good stereoacuity. Two were the authors (R.B. and R.O'S.), who are highly experienced at reporting binocular rivalry; the third (M.J.) was naive as to the purposes of the experiment.

Apparatus. Stimuli were generated by conventional TV techniques on the faces of two matched Tektronix 608 cathode-ray tube monitors (CRTs) with P31 phosphor. Gratings were produced by synchronously modulating the raster with sinusoidal waveforms from Clarke-Hess oscillators. The spatial frequency of the gratings was fixed at 4 cycles per degree (cpd). Gratings were flickered in counterphase by sinusoidally modulating the contrast of the grat- 
ings with the outputs of two other Clarke-Hess oscillators. Temporal modulation of uncontoured screens (i.e., full-field flicker) was effected by unsynchronously modulating the rasters at the desired temporal frequency. Outputs of the oscillators also could be combined with a linear summing amplifier and shown on both CRTs to achieve binocular presentation. With both gratings and full-field flicker, average luminance remained constant over space and time. All signals to the CRTs were first passed through HewlettPackard 350D attenuators, which allowed modulation depth (i.e., contrast) to be equated for all conditions.

The CRT screens were masked to expose a central circular area of $1.2^{\circ}$ at a viewing distance of $94 \mathrm{~cm}$. A mask consisted of a concentric dark rectangle $\left(4.02^{\circ} \times 3.29^{\circ}\right)$ centered within a larger light area $\left(7.73^{\circ} \times 6.33^{\circ}\right)$. The matched vertical contours provided by the two masks aided binocular alignment of the stimuli when viewed through a mirror stereoscope. Subjects corrected for any phoria by rotating the mirrors of the stereoscope, or by inserting a Risley prism in its eyepiece.

The subjects recorded binocular rivalry by pressing one of two buttons for periods of exclusive visibility of the stimuli. Button states were timed by a DEC LSI $11 / 23$ + minicomputer to within $1 \mathrm{msec}$ accuracy.

The apparatus was contained in a darkened viewing booth, with indirect lighting of the masks. Luminances were measured through the eyepiece with a Minolta spot photometer. The space average luminance of the CRT displays was $13.91 \mathrm{~cd} / \mathrm{m}^{2}$ with a spatial or temporal contrast of .30 . The dark parts of the masks reflected $0.04 \mathrm{~cd} / \mathrm{m}^{2}$, and the light parts $0.43 \mathrm{~cd} / \mathrm{m}^{2}$.

Procedure. The subjects initiated a trial by pressing a toggle switch. Events of a trial were as follows: blank screens for $5 \mathrm{sec}$; the left-eye field for $5 \mathrm{sec}$; the right-eye field for $5 \mathrm{sec}$; then both fields for $70 \mathrm{sec}$. The first $10 \mathrm{sec}$ of dichoptic presentation served as a warm-up period; rivalry recording began at the sound of a tone. A tone also signaled the end of the trial, at which time the screens became blank. At least 60 sec elapsed before the next trial.

At low temporal frequencies $(0.5$ and $1 \mathrm{~Hz})$, counterphase flicker was evident as a regular appearance and disappearance of the grating. Subjects were instructed to keep the appropriate button down for as long as that grating was completely suppressed. Of course, they were to release the button if parts or all of the other rival grating became visible.

Trials were run in blocks of 5 for uncontoured stimuli and 6 for temporally modulated orthogonal gratings, making 11 trials per session. Order of trials within blocks was random, and order of blocks was alternated over sessions. In each session, one eye viewed the standard temporal modulation of $4 \mathrm{~Hz}$ throughout. The eye receiving the standard and the orientation of the grating viewed by that eye were randomized between sessions, so that these two factors were counterbalanced. After four sessions, it became evident that very little rivalry was being reported when uncontoured stimuli differed only in temporal frequency. Accordingly, these trials were dropped for the remaining four sessions.

\section{Results and Discussion}

Full-field flicker. Dichoptic temporal frequency differences between uncontoured stimuli produced very little rivalry. This is illustrated in Figure 1, in which the cumulative time of buttonpresses, and hence dominance, is plotted against temporal frequency viewed by one eye while the other eye viewed $4 \mathrm{~Hz}$. Subjects described the combined display as flickering at an irregular temporal frequency intermediate between the monocular components. Sometimes "visual beats" were evident; these were probably due to the fact that, because of manual adjustment, dichoptic temporal frequency differences were not exact integer multiples. Most of the "rivalry" was

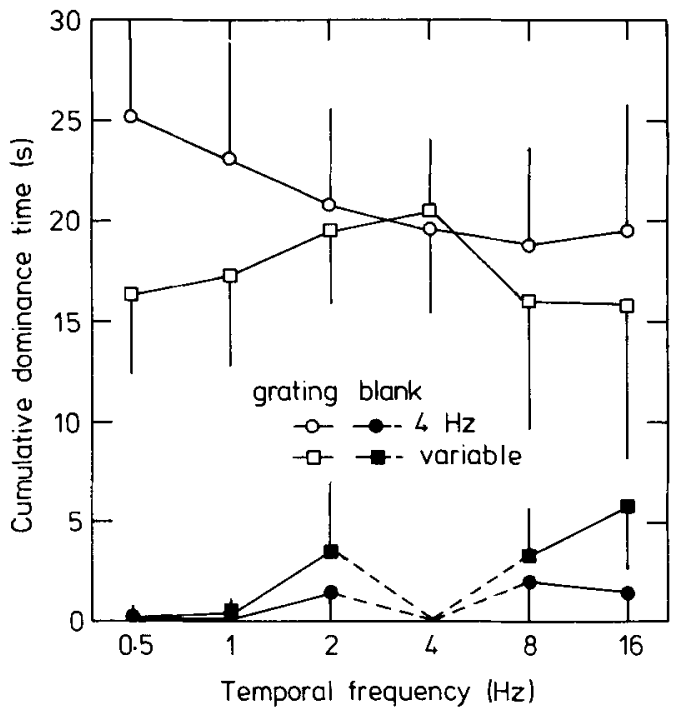

Figure 1. Cumulative time of exclusive visibility of each rival stimulus, plotted against the temporal frequency of the variable field. For full-field flicker (blank; i.e., filled symbols), each point represents the mean of 12 observations ( 3 subjects $\times 4$ trials). No observations were made with a variable temporal frequency of $4 \mathrm{~Hz}$, because this is a condition of zero temporal-frequency disparity; dashed lines join the functions to zero at this value. For phase-shifting gratings (grating; i.e., unfilled symbols), each point represents the mean of 24 observations. Vertical bars show standard errors.

reported by the naive subject, who was nevertheless unsure about whether she was actually experiencing rivalry.

To examine further the perceptual outcome under these conditions of full-field flicker, we compared dichoptic presentation with binocular viewing of the electronic combination of the component frequencies. In the latter condition, the two eyes viewed identical targets composed of the linear addition of two rates of flicker. The naive subject was shown, in a random order, all combinations of binocular and dichoptic presentation with the various temporal frequency conditions. She was asked to determine the nature of the presentation while keeping both eyes open, to describe her percept, and to record any rivalry she experienced.

Her accuracy at discriminating binocular from dichoptic presentation was at chance level. This is not to say that the discrimination could not be learned, but it shows that percepts from the two forms of presentation were phenomenally similar, which is not the case with binocular presentation of a grid versus dichoptic presentation of orthogonal gratings (Wade, 1975).

The naive subject reported very little rivalry, noting that the presence of "catch trials" had altered her response criterion. She experienced "rivalry" with binocular superimposition of 4- and $16-\mathrm{Hz}$ flicker; her percept was similar in character to that from the same components dichoptically presented. ' Her percepts usually were described as irregular visual beats or irregular changes in apparent temporal frequency. One of us (R.O'S.) com- 
pared binocular and dichoptic presentation with the same components over the full range of temporal frequencies. At low frequencies of the variable component, the two modes of presentation resulted in very similar percepts. At higher frequencies, the visual beats evident from binocular presentation were more regular than those from dichoptic presentation.

From the above observations, we conclude that dichoptic presentation of different temporal frequency components produces percepts similar to, but not exactly the same as, percepts produced by their monocular superimposition. Certainly, the experience is unlike that produced by conventional contour rivalry. The question of the nature of alternations reported from temporal frequency differences between uncontoured stimuli was further addressed in Experiment 2.

Phase-shifted gratings. Rivalry between orthogonally oriented sine-wave gratings was clear-cut and seemed to present fewer criterion problems for subjects than there were with full-field flicker. Figure 1 shows the significant interaction between temporal frequency of the variable and 4 - $\mathrm{Hz}$ fields $[\mathrm{F}(4,8)=5.54, \mathrm{p}<.05]$. One grating or the other was exclusively visible for about $65 \%$ of the time at all temporal frequencies. For rates below $4 \mathrm{~Hz}$, the more slowly flickering grating was seen for less time than the standard $4-\mathrm{Hz}$ grating. At and above $4 \mathrm{~Hz}$, there was no reliable difference in the relative dominance of the gratings.

The temporal-frequency dependence described above could be explained in terms of stimulus strength (Levelt, 1968) of flickering gratings. Changes in flicker rate will alter the position of a stimulus on the spatiotemporal contrast sensitivity surface (Kelly, 1977). Apparent contrast will decrease for flicker rates lower than about $4 \mathrm{~Hz}$, and hence such stimuli should dominate for less time (Blake, 1977). Nevertheless, there are some difficulties with this explanation. First, there should be an equivalent reduction in dominance for gratings flickering faster than $8 \mathrm{~Hz}$, and a reciprocal increase for the 4-Hz field; this is not apparent in Experiment 1. Second, actual variations in stimulus strength produce reliable changes in other measures of rivalry, such as alternation rate and mean period of dominance (Levelt, 1968). Rate- and period-temporalfrequency functions found here do resemble those predicted, but with large standard errors. Third, there were significant individual differences in rate- and periodtemporal-frequency functions in Experiment 1 (these were the cause of the large standard errors). In Experiment 2, we used a different dependent measure, in the hope that it would minimize the individual differences.

\section{EXPERIMENT 2}

In Experiment 1, subjects reported difficulties in deciding whether alternations experienced during dichoptic presentation of full-field flicker constituted rivalry. Also, there were large individual differences in rivalry reports from counterphase-flickering contoured stimuli. It was not clear whether subjects' reports reflected different visual

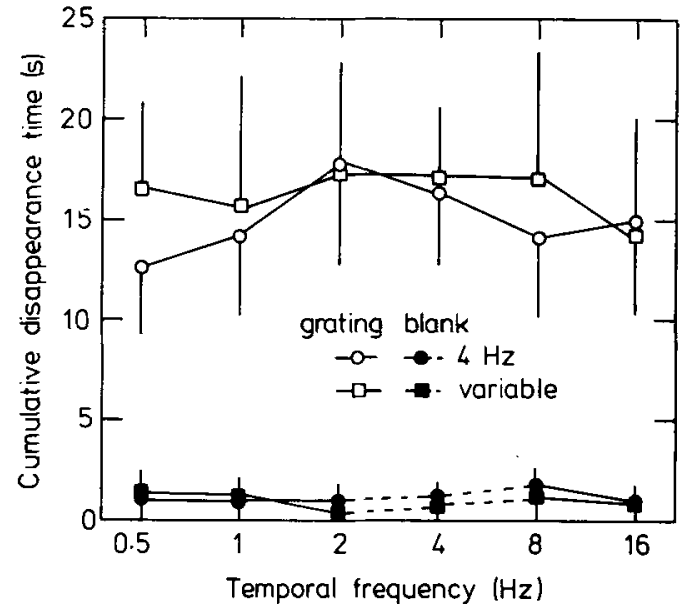

Figure 2. Cumulative time of disappearance of markers superimposed on each rival stimulus, plotted against the temporal frequency of the variable field. Unlike in Experiment 1, measures could be taken at zero temporal-frequency disparity; these points at $4 \mathrm{~Hz}$ represent the control for Troxler's fading, and are joined to the others by dashed lines. Each point represents the mean of 12 observations ( 3 subjects $\times 4$ trials). Vertical bars show standard errors.

processing or were due to different response criteria employed. Hence, it seemed prudent to search for a rivalry measure that was less susceptible to response criteria. We decided to use the approach of Smith, Levi, Manny, Harwerth, and White (1985), who tagged each rival field with a small monocular identifying marker and required subjects to report disappearances of the markers.

\section{Method}

The subjects and apparatus were the same as in Experiment 1, with the addition of monocular markers to each field. The left-eye field contained a circular spot $11^{\prime}$ in diameter, the center of which was $18^{\prime}$ directly below the center of the field. The right-eye field contained a similar marker located $18^{\prime}$ above center. Each marker reduced the luminance of the display at that point by $35 \%$. The procedure was the same as in Experiment 1 except for the following: Subjects pressed the left button for disappearance of the lower (left-eye) marker, and the right for disappearance of the upper; they were asked to note any occasion when both markers disappeared; there was no left- or right-eye preview of the rival stimuli; a condition was included in which $4-\mathrm{Hz}$ full-field flicker was presented to both eyes (to assess Troxler's fading of the markers); and data were collected over four sessions for all stimulus conditions.

\section{Results and Discussion}

Full-field flicker. Figure 2 shows cumulative time of marker disappearances plotted against temporal frequency. During full-field flicker, both markers were visible for about $95 \%$ of the time. Disappearances were rare and quite brief. Simultaneous disappearance of both markers was even more rare. There seems to be no relationship between the disappearances and the component temporal frequencies. Note that at $4 \mathrm{~Hz}$, there was no temporal disparity between the two fields, and yet there was the same amount of disappearance of the markers as when disparity was present. We believe that these disap- 
pearances with full-field flicker are examples of Troxler's effect and do not indicate disappearances from binocular rivalry suppression of the field on which the marker was superimposed.

Phase-shifted gratings. When the markers were superimposed on dichoptically presented orthogonal phaseshifting gratings, disappearances were frequent and had long average durations. This is shown in Figure 2 as a more-than-50\% cumulative time of disappearance. A three-way within-subjects analysis of variance found the difference between full-field flicker and phase-shifting gratings to be the only significant effect $[F(1,2)=659.82$, $\mathrm{p}<.01]$.

The use of markers minimized the individual differences in the shapes of the functions relating various rivalry measures to temporal frequency. The markers disappeared and returned abruptly, apparently in concert with the rivalry alternations. However, both markers were visible close to $50 \%$ of the time, longer than would be expected on the basis of the cumulative duration of visibility of composites found in Experiment 1 (about 35\%). Also, the significant interaction between temporal frequency of the variable and 4- $\mathrm{Hz}$ fields found in Experiment 1 is not evident in the results with markers. The use of markers to solve criterion problems apparently introduces other problems of a Heisenbergian nature; that is, the markers provide nonrival, stationary contours which may interact with the rival fields to alter rivalry. The exact nature of the alteration is not clear and is the subject of further research (in progress).

What can be said unequivocally is that rivalry between the contoured stimuli produced disappearances of the markers. The virtual absence of such disappearances when only a temporal disparity was presented (full-field flicker) suggests that conventional rivalry does not occur under those conditions. In this important respect, the results of Experiment 2 confirm and clarify the results of Experiment 1.

\section{GENERAL DISCUSSION}

Two findings from Experiments 1 and 2 need to be explained. First, flicker influences binocular contour rivalry. Second, and critically, dichoptic differences in sinusoidal flicker in the absence of contour do not produce rivalry.

Several explanations may be proposed for the influence of flicker on contour rivalry. Flicker could influence the rivalry mechanism itself. Indeed, earlier work has shown that the incidence of rivalry can be reduced (O'Shea \& Crassini, 1984) or completely abolished ("abnormal fusion"; Wolfe, 1983) when rival targets are briefly, repetitively flashed. However, the stimulus conditions we employed were quite different from those used in these earlier studies. Rather than using on/off flicker, we phase-shifted the gratings at temporal frequency and phase values yielding normal rivalry (O'Shea \& Crassini, 1984). Therefore, we are disinclined to believe that flicker affected the rivalry mechanism itself in the condition involving contour rivalry. It seems more plausible, as suggested earlier, that counterphase flicker influenced the apparent contrast of the contours, thus changing a rival grating's stimulus strength (Levelt, 1968).

The failure of pure flicker disparity to induce rivalry is more difficult to explain, especially because interocular inhibitory effects have been found in studies comparing monocular and binocular perception of flicker (see Blake \& Fox, 1973, for a review of this literature). In general, the critical temporal frequency at which perception of flicker is lost (CFF) is higher for binocular than for monocular viewing when the two eyes view equal rates of flicker presented in phase to the two eyes; this finding is referred to as "binocular summation." However, when the two eyes receive equal rates of flicker $180^{\circ}$ out of phase, binocular CFF is significantly lower than monocular CFF. The latter result could be interpreted as evidence for binocular suppression in the case of out-of-phase flicker. To test this hypothesis would require the use of some indirect assay of suppression (e.g., probe sensitivity; see Fox \& Check, 1966), which no study to date has employed. Furthermore, previous flicker studies involved presenting equal rates of flicker to the two eyes, whereas our emphasis has been upon conditions in which the two eyes view different temporal frequencies. Under these conditions, we have found no evidence for binocular suppression, even when we used an indirect method (i.e., the markers employed in Experiment 2).

One earlier study did use stimulus conditions more closely resembling those used here. Sherrington (1911) dichoptically presented different rates of on/off luminance modulation, usually in the ratio of $1: 2$, in his experiments on binocular perception of brightness and flicker. As an aside, he mentioned that rivalry was occasionally observed, but as this was not his main interest Sherrington did not elaborate on the specific conditions which produced rivalry. Consequently, it is impossible to know whether the temporal conditions he employed were comparable to ours. In any case, there are several differences between Sherrington's stimuli and ours that could explain his observations of rivalry. First, on/off, square-wave flicker is a highly complex stimulus, compared with the sinusoidal modulation we used. A second difference, related to the first, is a possible confounding of brightness and flicker in Sherrington's experiments. Sherrington himself noted that brightness is related to the rate of on/off flicker; the rivalry he observed may have been determined by brightness disparity rather than by flicker per se. Certainly, sinusoidal luminance modulation around a mean value and within the linear ranges allowed by our CRT screens did not produce any evident overall brightness differences related to flicker rate.

Under the conditions of our experiments, temporal frequency differences in full-field flicker did not lead to binocular rivalry. Instead, the subjects perceived either binocular beats or a temporal frequency intermediate between the two flicker rates. The latter observation is simi- 
lar to one made by Oguchi, Katsumi, and Kawara (1982), who dichoptically presented identical checkerboards that phase-shifted at different rates. They found that subjects reported an apparent temporal frequency different from those actually presented. Fourier analysis of the frequency of EEG potentials evoked by that display revealed a component at the intermediate frequency, as well as components equal to the stimulation rates. Katsumi, Peli, Oguchi, and Kawara (1985) confirmed this and showed that a realizable neural network could accomplish such frequency fusion. Our observation of binocular temporal beats is noteworthy in that such beats are not seen when different spatial frequencies are viewed dichoptically (e.g., Blakemore, 1970). Under the latter conditions, observers experience stereoscopic tilt or binocular rivalry, depending on the magnitude of the disparity in spatial frequency. This further underscores that the binocular visual system treats interocular temporal and spatial disparities differently.

The failure of dichoptic temporal-frequency differences to induce rivalry has important implications for models of rivalry that incorporate temporally tuned mechanisms. Hollins (1980) found that the spatial frequency of orthogonal dichoptic gratings that produced the most rivalry ( 3 cpd) was coarser than the peak of the contrast sensitivity function (around $5 \mathrm{cpd}$ ). To account for this, he proposed that transient mechanisms influence rivalry, and he speculated that dichoptic disparities in temporal frequency, "visible" only to transient mechanisms, would cause rivalry. Our results suggest that this notion is incorrect.

If flicker is considered as a way of exciting particular analytic visual mechanisms, it may seem surprising that flicker plays such a minor role in rivalry. When considered in ecological terms, however, this minor role makes sense. Under natural viewing conditions, interocular differences in form or motion may arise at any point in the visual field outside Panum's fusional areas. Rivalry can be thought of as the process that deals with such form or motion disparity. In the case of flicker, however, it is difficult to think of any situation that would produce dichoptic temporal-frequency differences. Hence, it is unlikely that the binocular visual system needs to be geared to deal with dichoptic differences in flicker.

\section{REFERENCES}

BLAKE, R. (1977). Threshold conditions for binocular rivalry, Joumal of Experimental Psychology: Human Perception \& Performance, 3, 251-257.

BLAKE, R., \& Fox, R. (1973). The psychophysical inquiry into binocular summation. Perception \& Psychophysics, 14, 161-185.
Blake, R., Zimba, L., \& Williams. D. (1985). Visual motion, binocular correspondence and binocular rivalry. Biological Cybernetics, 52, 391-397.

Blakemore, C. (1970). A new kind of stereoscopic vision. Vision Research, 10, 1181-1199.

Breese, B. B. (1899). On inhibition. Psychological Monographs, 3 , $1-65$.

Fox, R., \& CHEck, R. (1966). Forced-choice form recognition during binocular rivalry. Psychonomic Science, 6, 471-472.

Fox, R., Todd, S., \& Bettinger, L. A. (1975). Optokinetic nystagmus as an objective indicator of binocular rivalry. Vision Research, 15, 849-853.

Hollins, M. (1980). The effect of contrast on the completeness of binocular rivalry suppression. Perception \& Psychophysics, 27, 550-556.

Hollins, M., \& LEUNG, E. H. L. (1978). The influence of color on binocular rivalry. In J. C. Armington, J. Kraushopf, \& B. R. Wooten (Eds.), Visual psychophysics and physiology. New York: Academic Press.

Katsumi, O., Peli, E., Oguchi, Y., \& Kawara, T. (1985). Effect of contrast on fusional visual evoked potential (VEP): A model and experimental results. American Journal of Optometry \& Physiological Optics, 62, 233-239.

KeLLy, D. H. (1977). Visual contrast sensitivity. Optica Acta, 24, 107-129.

Kulikowski, J. J., \& Tolmurst, D. J. (1973). Psychophysical evidence for sustained and transient detectors in human vision. Journal of Physiology, 232, 149-162.

LeVelt, W. J. M. (1968). On binocular rivalry. The Hague: Mouton.

Oguchi, Y., Katsumi, O., \& Kawara, T. (1982). Binocular VECP with and without fusion. Documenta Ophthalmologica, 31, 415-420.

O'SHEA, R. P., \& CRASSINI, B. (1984). Binocular rivalry occurs without simultaneous presentation of rival stimuli. Perception \& Psychophysics, 36, 266-276.

SHERRINGTON, C. S. (1911). The integrative action of the nervous system. London: Constable.

SMith, E. L., III., Levi, D. M., ManNy, R. E., Harwerth, R. S., \& WHITE, J. (1985). The relationship between binocular rivalry and strabismic suppression. Investigative Ophthalmology \& Visual Science, 26, 80-87.

WADE, N. J. (1975). Monocular and binocular rivalry between contours. Perception, 4, 85-95.

Wade, N. J., De Weert, C. M. M., \& Swanston, M. T. (1984). Binocular rivalry with moving patterns. Perception \& Psychophysics, 35, $111-122$.

WALLACH, H., \& ADAMS, P. A. (1954). Binocular rivalry of achromatic colors. American Journal of Psychology, 67, 513-516.

WoLFE, J. M. (1983). Influence of spatial frequency, luminance, and duration on binocular rivalry and abnormal fusion of briefly presented dichoptic stimuli. Perception, 12, 447-456.

\section{NOTE}

1. The possibility cannot be excluded that the subject was experiencing monocular rivalry with binocular presentation.

(Manuscript received September 13, 1985; revision accepted for publication November 27,1985 .) 